\title{
La géographie économique à l'épreuve de la mondialisation
}

\author{
Economic Geography \\ confronted with Globalization
}

\author{
Solange Montagné Villette
}

Université Paris 13 CRESC

\begin{abstract}
Résumé
la financiarisation et la mondialisation de l'économie modifient profondément la géographie économique. Après avoir vu comment l'hypermobilité spatiale et structurelle des entreprises s'accorde mal avec le temps scientifique, on s'attache à relire les anciens paradigmes à la lueur des bouleversements récents et à définir de nouvelles pistes de recherche concernant plus spécifiquement le travail. On montre ensuite combien les nouveaux espaces productifs asiatiques, loin de se cantonner aux activités à faible valeur ajoutée, s'orientent vers la technologie et se présentent, dans certains cas comme des concurrents des pays industrialisés. Enfin il convient de réexaminer les rapports Nord-Sud, en montrant qu'apparaît un sous-développement social en grappes.
\end{abstract}

(c) 2008 Lavoisier, Paris. Tous droits réservés.

\section{Summary}

finance and globalisation are deeply transforming economic geography. After seeing how spatial and structural hypermobilities are the opposite of scientific time, we shall try to re-read the old paradigms in the light by the recent bouleversements distresses and to describe in particular new ways of researching the world of work. In a second part we will show that the new Asiatic and productive spaces, far from being confined to low added value production are now developing in new technologies in the hope of competing with industrialised countries. Finally focusing on the new North-South borders, we shall show that the arrival of spaces of social underdevelopment are diffused in the North and the South.

(C) 2008 Lavoisier, Paris. Tous droits réservés.

*Adresse email : solange.villette@orange.fr

doi:10.3166/ges.10.451-468 @ 2008 Lavoisier, Paris. Tous droits réservés. 
Mots clés : géographie économique, hypermobilité, éclatement spatio-temporel du travail, espaces productifs, protectionnisme, libéralisme, sous-développement social

Keyswords: economic geography, hypermobility, the breakdown of the spatio-temporal dimensions of work, productive spaces, liberalism, protectionism, social underdevelopment

La mondialisation des activités et l'importance croissante de la financiarisation ont pour conséquence un changement de finalité productive et une dilatation de l'écoumène économique. Ce double changement conduit, depuis une vingtaine d'années, à une autre géographie de l'entreprise et du travail, ainsi qu'à une forme de rééquilibrage Nord-Sud. Après avoir plus particulièrement étudié les hypermobilités spatiale, structurelle et financière des activités, qui constituent quelques-uns des nouveaux paradigmes remettant en cause la manière d'aborder la recherche en ce domaine, on en examinera les conséquences. En effet, ces changements brutaux de l'espace et du temps des entreprises conduisent à réviser ou relire les anciennes thématiques, à la lumière des faits, et à explorer de nouvelles pistes de travail pour le géographe. Enfin, l'apparition, également très rapide de nouveaux espaces productifs, notamment en Asie, remet en cause la hiérarchie des puissances économiques, ainsi que la formulation de la géographie du sous-développement.

\section{Financiarisation et hypermobilité des activités}

La recherche systématique des prix et des contraintes les plus faibles a pour conséquence l'hypermobilité spatiale et structurelle de l'activité et des entreprises, dont on constate de plus en plus fréquemment le comportement erratique. Il en résulte pour le géographe, qui travaille dans le temps présent, des difficultés croissantes à étudier avec recul (temps scientifique), des phénomènes qui changent parfois au jour le jour.

\subsection{La poursuite du moins-disant et ses contradictions}

L'un des moteurs de l'économie actuelle est la recherche du moins-disant en matière de prix, de salaires, d'investissements, mais aussi de règles environnementales et sociales. Cette finalité, inscrite dans une spirale descendante, trouve ses limites dans l'irrationalité et les contradictions entre court et long termes. En effet, si, à court terme, l'intérêt particulier de l'entreprise est de produire ou d'acheter moins cher dans les pays émergents, si celui du consommateur est de payer ses produits bon marché, l'intérêt général est autre. À long terme, la collectivité n'y trouve pas son compte pour des raisons écologiques, économiques et sociales. Sur le plan écologique, tout d'abord, il est contradictoire d'encourager le « développement durable » tout en transportant une multitude de produits, notamment des pondéreux, d'un bout à l'autre de la planète. Remplir des bateaux de granite chinois, à destination de la Bretagne (Rennes, Landerneau, Daoulas) ou du Canada (Montréal) pour les besoins de la voirie locale, à la seule raison qu'il est moins cher à l'achat, laisse perplexe quant aux économies supposées. D'autant qu'il existe sur place des carrières de qualité. Si la compétitivité du granite chinois est indiscutable, d'autres éléments entrent en ligne de compte. À échelle locale, si une collectivité territoriale y trouve momentanément un allé- 
gement financier, elle peut voir aussi son nombre de chômeurs augmenter. De même, si la région chinoise productrice s'enrichit en exportant, c'est souvent au prix de conditions de travail pénibles et d'une exploitation inconsidérée des ressources naturelles. À échelle mondiale, le bilan est tout aussi discutable : l'augmentation des échanges commerciaux de ce type consomme une quantité d'énergie considérable, notamment du pétrole, dont on sait qu'il est en voie de disparition; la pollution s'accroît. Le développement économique chinois, symbole du développement du Sud, pour justifié qu'il soit, doit-il impliquer un gaspillage des ressources non renouvelables ? Doit-il passer, à la fois, par l'exploitation de la main-d'œuvre locale et l'augmentation des déséquilibres écologiques, dont on suspecte les dangers sur la santé de la population mondiale?

Le déplacement incessant de matières premières, de produits ou de main-d'œuvre à la recherche du moins-disant, peut aussi avoir lieu à l'intérieur d'un même ensemble économique et donner lieu à des trajets aberrants. Que dire des déplacements agroalimentaires? La fabrication d'un yaourt aux fraises nécessite en moyenne $9000 \mathrm{~km}$ de trajet en Europe, soit $40 \mathrm{~g}$ de pétrole au $\mathrm{kg}$. Que dire du trajet des pommes de terres néerlandaises, épluchées en Italie, avant d'être transformées en frites dans une usine belge? L'idée développée par A. Lebeau (2005) que « nous sommes la première génération à être confrontée, par le développement de la technique, aux limites de l'espace et des ressources de notre planète » et que « nous ne sommes pas capables de penser véritablement que, dans ces conditions, l'extinction de l'espèce humaine est possible » n'a pas encore fait son chemin.

Sur le plan économique, la géographie ne peut que constater le même type de contradictions. Les délocalisations au sens large du terme, c'est-à-dire le transfert des fabrications ou des services, relevant de la seule recherche du moindre coût et non pas d'une technologie particulière ou d'une innovation, créent des emplois et de la richesse dans des pays émergents au prix d'un moins-disant salarial, social et environnemental que désirent fuir les populations locales en émigrant vers les pays riches. Là encore, l'intérêt individuel ou régional s'oppose à l'intérêt général. Quelle rationalité dans ce chassé-croisé des emplois, des capitaux et des hommes? Certes, l'entreprise réalise sur le court terme des profits plus importants. Sur le long terme, on perçoit deux types de déconvenues. D'une part un rétrécissement du marché des pays riches, en raison de l'appauvrissement dû à la diminution des emplois. D'autre part, des coûts sociaux du travail de plus en plus élevés dans la mesure où c'est justement la forte protection sociale qui attire les migrants du Sud vers le Nord. En effet, bien des migrants récents persistent dans leur projet, même s'ils savent qu'ils trouveront difficilement du travail, et ce pour bénéficier d'une protection sociale, sanitaire et de meilleures conditions démocratiques (rapport sur les politiques migratoires 2006).

Autrement dit, il y a actuellement déconnexion totale entre la localisation idéale de l'activité et celle du salarié ce qui génère des flux et migrations inversés et contradictoires. Contrairement aux délocalisations des années 1980, celles des années 2000 créent de moins en moins d'emplois qualifiés au Nord, y compris pour les cadres. Or la diminution des emplois du Nord induit une forte demande de protection sociale et le renchérissement des coûts salariaux. Cette protection, jusqu'à présent croissante en Europe de l'Ouest (salaire minimum, allocation chômage, retraite, couverture santé, revenu minimum, allocation d'inaptitude), attire la main-d'œuvre des pays pauvres, au moment où elle est revue à la baisse. Le Nord n'est plus à même d'offrir les mêmes aménités; la protection s'amenuise (âge de la retraite), le salarié se précarise (travail intermittent)... L'information 
passe d'autant plus mal auprès des pays du Sud que les politiques minimisent la situation. Il en résulte d'importantes frustrations, notamment pour les migrants, voire des révoltes sociales, pouvant prendre des formes culturelles.

Certes, l'irrationalité n'est pas nouvelle, mais la mondialisation a agrandi spatialement l'écoumène économique en même temps qu'elle rétrécissait les temps de parcours des ressources, des biens, des personnes, des capitaux et de l'information grâce à l'extraordinaire amélioration du transport. L'espace-temps de la géographie a changé. La cohérence qui liait spatialement activité et richesse (FIG 2005) s'efface progressivement. Sur le plan épistémologique, cela se traduit actuellement par une difficulté à élaborer des concepts pérennes et des modèles de localisation, à définir des espaces de gouvernance ou d'intervention pertinents. L'hypermobilité remet en cause les paradigmes de la localisation des activités

\subsection{L'hypermobilité spatiale ou l'entreprise « dromomane »}

Dès 1986, B. Dezert évoquait la segmentation de la production par la sous-traitance et les stratégies de mobilité : « la grande usine à lourds investissements (bâtiment), à amortissements de longue durée, est désormais obsolète ». G. Benko (1986) signalait que «l'industrie de haute technologie est considérée comme mobile, en regard des facteurs de localisation traditionnelle », et insistait notamment sur le rôle du cycle de produit, extrêmement court, dans cette activité. La mobilité croît en intensité et dans tous les domaines. Le cycle de vie d'un «bien durable de consommation électrique » évalué à 20 ans, s'est réduit à quelques années (téléviseur). Les produits à cycle très court, spécifiques du prêt-à-porter (Montagné Villette 1987), s'étendent à tous les domaines. Les géographes perçoivent déjà l'hypermobilité, même s'ils ne la théorisent pas encore sous sa forme actuelle. Actuellement, la mobilité des industries de haute technologie gagne tous les secteurs, y compris celui des services. L'information, la gestion personnalisée d'opérations bancaires, l'analyse des données médicales, le commerce, y compris le commerce alimentaire, le paiement sont autant de services qui peuvent être transférés loin de l'usager, parfois à des centaines, voire des milliers de kilomètres. Dans certains cas l'entreprise de services, spatialement dépendante des coûts de main-d'œuvre, entraîne la mobilité de l'usager lui-même. Ainsi en est-il de certaines opérations médicales, esthétiques, dentaires, etc., pratiquées en Thaïlande, en Inde à moindre prix. Ainsi en est-il aussi, pour l'accueil des personnes âgées dans des structures médicalisées ou des résidences de services du troisième âge en Tunisie. Cette dernière tendance, pour limitée qu'elle soit encore, prendra vraisemblablement de l'importance, compte tenu du vieillissement de la population européenne et des coûts qui en découlent.

En matière de géographie économique et industrielle, la mobilité ne constitue donc pas une nouveauté. Néanmoins, la notion a, depuis les débuts de la géographie institutionnelle, plusieurs fois évolué en fonction de différents paramètres. La localisation des matières premières, de l'énergie, de la main-d'œuvre, du marché, des places financières a constitué, à différentes époques, les références indispensables à l'étude de la mobilité. Manzagol (1980), Mérenne-Schoumaker (1991) qui analysaient avec rigueur les facteurs de localisation, évoquaient de nouveaux espaces productifs (littoraux, technopôles, NPI...). Mais ils ne pouvaient prévoir l'accélération due à la révolution informatique et des transports. Or, depuis une vingtaine d'années, c'est justement cette révolution 
de l'instantané qui complexifie la situation. En passant du changement de localisation justifié par un changement de paramètres, à des changements successifs et rapprochés, visant une simple augmentation de bénéfices à très court terme, on est passé de la mobilité à l'hypermobilité. On qualifiera donc ici d'hypermobile, une entreprise dont les changements successifs de localisation répondent avant tout à des impératifs financiers. L'hypermobilité induit une dépendance accrue vis-à-vis d'autres territoires aux qualités éphémères. Les constructeurs automobiles de l'Europe occidentale, transférant en 2008 leurs activités vers la zone dollar, à la seule raison de la faiblesse actuelle de celui-ci, s'exposent au risque d'une remontée des taux de cette devise. Ce transfert peut offrir des bénéfices supplémentaires, mais non l'assurance d'une stabilité des salaires (Pottier 2003) permettant de compenser les coûts directs et indirects induits. Les outils de la mondialisation ont rendu l'entreprise « dromomane ${ }^{1} »$.

Désormais l'entreprise, surtout l'usine, ne constitue plus un point d'ancrage permanent ou semi-permanent, une sorte de donnée territoriale permettant une lecture géographique de l'activité. Le cas de la petite entreprise «fantôme » du prêt-à-porter déménageant son matériel en une nuit (Montagné Villette 1990) pouvait sembler anecdotique et spécifique du circuit productif court. Or, le phénomène s'est étendu, sous des formes voisines, à nombre de secteurs industriels, voire aux entreprises classiques, ainsi qu'aux secteurs des services. Le matériel de l'usine BRS (pièces automobiles) dans le Doubs a été retrouvé dans des camions à destination de la Slovaquie en février 2008, alors même que les salaires de janvier n'avaient pas été réglés. Déménagement, transfert de l'équipement de l'usine ou des bureaux en l'absence des salariés (week-end, vacances), fermeture brutale sans préavis avec impossibilité de trouver les responsables, voire les propriétaires... relèvent d'une démarche voisine, même en l'absence de délits caractérisés. L'hypermobilité conduit à la suppression de l'activité pérenne. La nouvelle entreprise, en quelque sorte «éphémère », brouille le rapport homme/territoire productif et conduit à une géographie où le temps joue le rôle principal. L'exemple de Deawoo est à cet égard significatif. L'entreprise attirée en 1992 en Lorraine, et soutenue par 1 milliard de fonds publics, prévoit la création de 1000 emplois sur 3 sites; elle annonce des fermetures en 1999 et quitte le terrain en 2002. Deux changements de localisation en 10 ans : l'entreprise est devenu dromomane.

Si la logique productive capitaliste classique répondait à une sorte de rationalité fondée sur un équilibre offre/demande, la logique financière actuelle, inspirée des marchés boursiers, s'appuie davantage sur les paradigmes, plus aléatoires, de la psychologie et du jeu. En effet, quand en l'absence de difficultés financières vitales, l'entreprise abandonne brutalement lieu de production, mais aussi main-d'œuvre et savoir-faire pour un site mieux-disant, sa mobilité spatiale répond plus à un jeu financier ou une mode de gestion (et non plus un mode) qu'à une nécessité; plus à un confort qu'à une obligation. C'est donc la finalité de la mobilité, plus que la mobilité en soi, qui pose question. Compte tenu de l'importance du capital investi, tant en matériel qu'en ressources humaines, il convient de s'interroger d'une part sur les motivations d'un tel transfert, d'autre part sur l'aide des collectivités territoriales ou des états. Quel est le niveau du ratio capital/bénéfice exigé justifiant le transfert? Jusqu'où peut aller l'entreprise dans cette voie sans mettre le marché en péril?

\footnotetext{
${ }^{1}$ La dromomanie est une affection psychiatrique morbide caractérisée par le besoin irrésistible de marcher, de courir, de se déplacer.
} 
En fait, le comportement spécifique, considéré comme « marginal », des entreprises artisanales du Sentier, dans les années 1980, s'est largement répandu. Ce qui constituait une adaptation des petites entreprises est maintenant adopté par nombre de grandes entreprises et appartient pour certains à une sorte de norme. L'adaptation et la réactivité au marché, qui correspondaient au temps de l'entreprise et à celui de l'homme, doivent répondre aujourd'hui à celui de l'informatique et donc à l'immédiat. On est passé de la vitesse à la précipitation, éliminant ainsi le temps de la réflexion. Plus que sa pertinence, c'est l'immédiateté de l'action qui devient importante.

\subsection{L'hypermobilité structurelle ou l'entreprise éphémère}

À l'hypermobilité spatiale, se superpose de plus en plus une mobilité structurelle de l'entreprise dont les structures productives et financières varient sur le très court terme au gré des rachats.

La complexité de la géographie économique résulte principalement de celle de l'actuelle économie de marché. La multiplication des fusions, l'éclatement des fabrications en divers sites, la déterritorialisation des services, l'externalisation (Bost, Delapierre 2007), rendent difficile l'étude des processus productifs sur le moyen et le long termes. En effet, la complexité des croisements et changements financiers donne des entreprises à géométrie variable. Ces configurations changeantes, modelables parfois « au mois le mois » par le jeu des OPA et des fusions, font de l'entreprise une structure éphémère. Ces structures éphémères, dont témoignent les fréquents changements de noms, ne conservent le plus souvent que la ou les marques phares, en tant que support du marché. Quelques exemples récents illustreront notre propos

Dans l' «affaire Arcelor » (2006), une entreprise européenne dont la santé était suffisamment bonne pour racheter le Canadien Dofasco et s'ouvrir l'accès de l'Amérique du Nord, on ne peut que constater la complexité des enjeux. Complexité économique d'abord. Après avoir réalisé une OPA qui avait fait d'elle le deuxième groupe mondial, Arcelor, dont les actions avaient progressé de plus de $50 \%$ en 15 mois, fut à son tour l'objet d'une OPA de Mittal, numéro 1 mondial. Arcelor, groupe public-privé européen, dont le siège est à Amsterdam, est donc devenu un groupe familial indien. On constate ici la justesse de vue de B. Dézert (1986). L'entreprise, construction à structures solides et évolutives est devenue un assemblage éphémère d'intérêts, une sorte de structure caoutchouteuse modelable. En externalisant ou vendant une part de ses activités, en même temps qu'elle en rachète d'autres et ailleurs, pour quelques mois, voire quelques semaines, l'entreprise est susceptible de changer de configuration sans autre objectif que l'augmentation des profits. Dans ce cas, où elle a pour but premier, non de produire, mais de faire « un coup », ses critères de localisation changent. Il convient de la considérer, non plus comme un site productif répondant à certains besoins, mais comme un outil financier répondant à d'autres logiques

Complexité géopolitique ensuite; le groupe indien Mittal, déjà enregistré aux PaysBas, travaillait dans 14 pays dont la plupart émergents; le succès de son OPA lui a permis de s'implanter dans 60 pays, dont beaucoup sont développés. La réussite de l'OPA a également mis à mal l'idéal européen, dans la mesure où le groupe Thyssen-Krupp qui a perdu la bataille boursière contre Arcelor, lors du rachat de Dofasco, fut, à certains 
moments, l'allié objectif du groupe indien. Cette alliance, a priori contraire aux intérêts politiques européens, et plus particulièrement à l'alliance franco-allemande, souligne, s'il en était besoin, la supériorité du pouvoir financier sur le politique. Ces changements invitent à une relecture géographique.

\section{Thématiques à relire et pistes à explorer}

En matière économique, la distinction secondaire et tertiaire, pourtant maintes fois remise en cause (Békouche et Damette 1993, Montagné Villette 2001), a la vie dure dans l'inconscient politique et stratégique des décideurs (et parfois celui des chercheurs). Dans certains domaines, la mesure des bouleversements en cours n'a pas été prise. Certains thèmes étudiés dans les années 80 , doivent être complétés : la distinction industries-services, le bassin d'emploi, les effets de l'activité sur la répartition spatiale des populations, voire la morphologie urbaine. Le chiffre d'affaires, les effectifs, la valeur ajoutée, les investissements ne suffisent plus à la compréhension des implantations. Au plan économique, le rôle de l'état s'effiloche. L'innovation, apparue comme la solution-miracle après la crise économique des années 70, ainsi que la conservation ou le développement des fonctions de conception, doivent être revus à l'aune des faits récents et de l'hypermobilité. La notion de travail, plus étudiée par les sociologues que par les géographes, mérite, quant à elle, une étude approfondie, notamment dans son cadre spatio-temporel.

\subsection{L'effacement progressif des Etats}

On aborde ainsi une des données fondamentales de la géographie économique actuelle : l'affaiblissement, voire la disparition des états, non en tant qu'instance de régulation, mais en tant qu'espace économique. Pour J. Fache (2005), le territoire des états s'efface devant celui des compétences et des savoirs. Si l'on excepte les états forts (Chine), on peut admettre que le pouvoir politique cède progressivement la place au pouvoir économique. Les conséquences ne sont pas minces en démocratie. En effet, la population élit les politiques dont elle espère des changements qu'ils ne sont plus en mesure de réaliser. Sauf retournement brutal, il s'agit d'un phénomène inéluctable puisque la mondialisation, qui supprime les frontières de l'entreprise, n'a pas encore effacé celle de l'état-nation. Les limites de l'intervention de l'état, calquées sur celles de son territoire, excluent la plus grande part du champ économique devenu international. L'interventionnisme économique des états trouve ici ses limites. Le concept de «patriotisme économique » évoqué sous différentes formes par la France, les Etats-Unis, l'Italie... a peu d'outils à sa disposition (politique monétaire, subventions) dans le cadre du libéralisme affiché. La nationalité entrepreneuriale n'a plus grande signification, comme le montre le cas français (tableau 1). De 1997 à 2005, la part du capital étranger dans les entreprises du CAC est passée de 33 à $47 \%$. Seule une hypothétique gouvernance économique supranationale, dont on imagine encore mal les modalités, redonnerait aux états un rôle plus important 
Tableau 1 : internationalisation de quelques entreprises françaises cotées en 2006

\begin{tabular}{|c|c|c|c|c|c|}
\hline $\begin{array}{c}\text { Entreprise } \\
\text { du CAC }\end{array}$ & $\begin{array}{c}\text { \% activité } \\
\text { à l'étranger }\end{array}$ & $\begin{array}{c}\text { Effectif } \\
\text { total }\end{array}$ & $\begin{array}{c}\text { Dont } \\
\% \text { étrangers }\end{array}$ & $\begin{array}{c}\text { Etrangers } \\
\text { dans } \\
\text { la direction }\end{array}$ & $\begin{array}{c}\% \text { actionnariat } \\
\text { sur le marché }\end{array}$ \\
\hline TOTAL & 81 & 111401 & 71 & $0 / 7$ & 83,4 \\
\hline $\begin{array}{c}\text { SANOFI } \\
\text { AVENTIS }\end{array}$ & 86 & 96439 & 71 & $6 / 22$ & 71,56 \\
\hline BNP PARIBAS & 45 & 110000 & 50 & $0 / 11$ & 13,5 \\
\hline L'OREAL & 88 & 52081 & 77 & $2 / 9 *$ & 44,70 \\
\hline LVMH & 83 & 56509 & 65 & $3 / 5 *$ & 85,42 \\
\hline CARREFOUR & 51 & 430695 & 69 & $0 / 12$ & - \\
\hline BOUYGUES & 27 & 115400 & 45 & $2 / 5$ & \\
\hline PUBLICIS & 89 & 36384 & 89 & $*$ PDG étranger \\
\hline
\end{tabular}

Quoi qu'il en soit, l'exacerbation de la mobilité spatiale et structurelle de l'entreprise et les changements qui en découlent, rendent son analyse difficile. En effet, l'intensité et la rapidité de la mobilité sont telles que leur étude appartient davantage au temps journalistique qu'au temps scientifique. Cette donnée constitue un des problèmes fondamentaux de la géographie économique actuelle qui ne peut s'appuyer sur des paradigmes permanents, du moins sur une durée permettant l'analyse. L'espace d'activité, en tant que lieu fixe est progressivement supplanté par l'espace mobile et informel des réseaux relationnels à géométrie variable. L'entreprise Gemplus, fondée en France en 1988, en est l'archétype via ses rachats successifs, ses changements de stratégies, de sites et son passage sous domination américaine et fonds de pension (Daviet 2005). On peut donc émettre / admettre l'hypothèse que cette hypermobilité, résultant de la financiarisation de l'économie, peut aussi avoir des répercussions obligeant à une remise en cause scientifique de la manière d'étudier le sujet. Le rapport temps-espace prend, dans cette optique, le pas sur le rapport homme-espace. La géographie de l'entreprise proposée par S. Daviet (2004) devrait permettre d'ouvrir de nouvelles pistes grâce à l'élaboration d'un cadre théorique. En effet, si l'histoire de l'entreprise se justifie plus que jamais, fût-elle chaotique, la géographie classique doit s'adapter à l'espace désormais mobile de l'entreprise; sa localisation, sa stratégie industrielle, ses capitaux constituent autant d'éléments devenus « fluides » dont l'étude doit être repensée.

\subsection{Avance technologique et savoir-faire}

L'antienne de la formation de haut niveau permettant de conserver les emplois qualifiés, ou les emplois de conception, dans les activités de haute technologie a vécu. D'une part, la division internationale du travail traditionnelle, consistant à transférer les fabrications ou l'assemblage pour garder les produits haut de gamme et la conception, ne résiste pas 
à l'analyse récente. La Chine, dont on expliquait en 1986 (Montagné Villette) qu'elle ne pouvait fabriquer de manière satisfaisante des produits de luxe, en raison d'un manque de savoir-faire qualitatif de la main-d'œuvre et d'un manque d'hygiène, a comblé son retard. Exportatrice de cuvettes en émail dans les années 80, la Chine se positionne 20 ans plus tard sur le marché mondial des téléphones portables, des PC et s'introduit sur celui de la recherche aéronautique. Airbus y a ouvert en 2005 un centre de conception et de recherche pour une partie des études de l'A 350 : 54 ingénieurs chinois ont été recrutés en quelques mois. Actuellement, c'est donc aussi bien les produits courants que la fabrication, voire la conception, des produits de haute technologie, qui sont désormais délocalisés. La haute valeur ajoutée et le savoir faire manuel, la conception, l'innovation (FIG 2005), qu'on imaginait garder en pays industrialisés, sont désormais largement mondialisés

L'innovation, qui dans les années 1980-90 concernait le Nord s'étend désormais dans certains pays du Sud. La modernisation, l'automatisation et l'augmentation de la productivité du Nord sont rattrapées par celles du Sud. Les usines Rouleau-Guichard (Montagné Villette 1987) de Toulouse qui réussissaient à rester compétitives sur le marché du sousvêtement, grâce à l'avance technologique, ont progressivement délocalisé leurs fabrications, notamment au Maghreb. Cependant, les causes du transfert restent confuses L'entreprise affirme qu'elle ne pouvait plus faire face à la concurrence. Les syndicats ripostent qu'il s'agit seulement d'une recherche de profits supplémentaires pour investir dans d'autres secteurs à haut rapport financier (hôtellerie).

L'avance technologique et le savoir-faire du Nord se trouvent maintenant aussi au Sud. Le Nord qui utilise désormais les chercheurs à bas salaires du Sud n'a plus les mêmes besoins en personnel qualifié. Actuellement en Europe, on manque davantage d'une main-d'œuvre locale à bon marché, pour des tâches ancillaires, que de cadres. La France par exemple manque de personnel dans le bâtiment, la restauration, l'hôtellerie, les services à la personne et compte parallèlement de nombreux chômeurs y compris parmi les diplômés de haut niveau (chercheurs). Si l'innovation, la formation, la recherche constituaient des sésames pour l'emploi, le chômage des jeunes diplômés serait plus faible. Alors qu'un poste de juriste provoque en moyenne 300 candidatures, on manque dans le même temps de maçons, de boulangers... Cet état de fait, qui remet en cause le discours politique traditionnel sur les besoins en main-d'œuvre et les qualifications, mériterait une étude spatialisée plus approfondie.

\subsection{L'éclatement spatial du travail}

Dans le processus industriel ancien, la division internationale du travail constituait la première marche de l'éclatement productif. Le deuxième niveau est apparu avec la séparation des fonctions de direction et de production. Les fonctions de direction sont les seules à rester en milieu urbain, tandis la production est exilée dans des espaces périphériques, moins coûteux en foncier et en main-d'œuvre. En France, par exemple, on parle dans les années 1960 de décentralisation, c'est-à-dire du transfert des activités productives de la région parisienne vers la province, et plus particulièrement vers les zones rurales. Cette décentralisation faite, sous la houlette de l'état, dans le cadre de l'aménagement du territoire a bénéficié des primes afférentes (PAT). Plus tard (années 1980), on évoquera la métropolisation de certaines activités, c'est-à-dire l'implantation des activités à forte 
valeur ajoutée dans les villes disposant d'un potentiel supérieur en matière de recherche, de desserte internationale et d'aménités diverses. Mais les délocalisations qui transfèrent les activités productives les moins rentables vers le Sud constituent le processus d'éclatement le plus marquant de cette époque.

Depuis une vingtaine d'années, le processus d'éclatement productif à grande échelle se manifeste par le développement de la sous-traitance, dans des secteurs aussi différents que ceux des industries de main-d'œuvre ou de haute technologie. Il se poursuit par l'externalisation de certaines fonctions, selon des modalités spécifiques et progressives. Il se termine par les délocalisations. La sous-traitance, quelles qu'en soient les formes, correspond à un transfert de charges de l'entreprise donneuse d'ordre vers l'entreprise preneuse d'ordre, établissant de facto une hiérarchie des structures productives. À l'origine, la sous-traitance productive pure, consistant à transférer tout ou partie des fabrications vers une autre entreprise, impliquait une relative proximité spatiale des entreprises concernées pour satisfaire le marché. Mais cet éclatement productif, occasionnel ou intermittent, est devenu progressivement permanent. $\mathrm{Au}$ Japon par exemple, il conduit à un système dual : grandes entreprises protectrices (législation sociale et environnementale) et petites entreprises avec salariés mal protégés.

En fait, le transfert productif se métamorphose rapidement en transfert socio-économique. Quand la production, les incertitudes économiques, les investissements, les obligations législatives sont transférés massivement vers des structures plus petites, l'entreprise donneuse d'ordre passe progressivement de l'industrie au commerce, de la production à la conception, voire la finance. La mondialisation, qui généralise soustraitance et délocalisation vers les pays émergents, a renforcé l'éclatement spatial du travail. La production dispersée entre plusieurs sites, puis plusieurs pays, a atteint une grande complexité. Déjà en 1985 l'étiquette des collants Tex (Carrefour) illustrait ce phénomène : «Assemblé à l'étranger, tricoté, teint et emballé en France, made in Rumania ». Quant aux automobiles européennes ou américaines, elles comportaient en moyenne, dès les années 90 , des pièces en provenance de 35 pays. Actuellement Renault se dit non plus constructeur, mais concepteur d'automobiles.

Néanmoins cet éclatement productif trouve parfois des limites spatiales comme le montrent les PIF (parcs industriels fournisseurs) de l'automobile (Gorgeux, Mathieu 2000). Si l'emploi est éclaté entre diverses entreprises, les contraintes spatiales reviennent en force notamment dans le cadre du juste-à-temps et de la suppression des stocks. Le donneur d'ordre regroupe alors ses sous-traitants à proximité immédiate, voire sur son propre terrain. L'usine automobile Smart de Hambach, créée en 1997, constitue un modèle en la matière (Montagné Villette 2005) : l'ensemble des sous-traitants est rassemblé sur un même lieu; les effectifs du constructeur sont inférieurs à ceux des sous-traitants qui doivent suivre exactement le rythme de la production. Le PIF permet à la fois externalisation et intégration de proximité. Le lien de dépendance et la division du travail s'inscrivent non plus dans le cadre Nord-Sud et l'espace-monde, mais dans le cadre local d'un espace commun. En matière d'emploi et de travail, les conséquences commencent tout juste à apparaître : il s'agit, ni plus ni moins, que de la juxtaposition de différents types de travail. Un modèle de travail classique de pays riche et un nouveau modèle de travail, pas encore totalement déterminé, qui s'apparente à celui des pays dépendants. Dans cette optique, le concept de Tiers-Monde fondé sur la croissance mérite une relecture plus sociale qu'on abordera ultérieurement. 


\subsection{L'emploi éclaté dans le temps}

Les impératifs économiques déjà évoqués ont transformé le travail dans sa globalité, mais aussi dans sa temporalité. Cette donnée concerne plus directement l'homme que l'entreprise. L'éclatement temporel du travail, analysé par rapport au modèle antérieur des Trente Glorieuses, pour sociologique qu'il paraisse au premier abord, n'en a pas moins des conséquences géographiques fondamentales.

Les grandes amplitudes de travail, qui selon la formule anglo-saxonne passent progressivement du 9-5 au 24-7 (c'est-à-dire d'une amplitude de travail de 8-9 heures par jour, 5 jours par semaine, à une amplitude de 24 heures, 7 jours sur 7), entraînent une répartition différente des activités. Le travail est non plus continu, mais séparé par de longues plages de temps libre/temps mort (Montagné Villette 2005). Il implique une multiplication des trajets domicile-travail, et donc soit un logement à proximité, soit un moyen de transport personnel, plus coûteux que les transports en commun. Quand on sait que ce travail éclaté concerne plus particulièrement les salariés les moins payés, et donc ceux qui ont peu de choix en matière de logement, on voit très vite les conséquences géographiques. Pareillement, l'irrégularité hebdomadaire découlant de l'annualisation disperse le travail en l'étalant sur la semaine, y compris les dimanches et certains jours fériés, désorganisant la vie du salarié, mais aussi en cascade les fréquentations touristiques, commerciales ainsi que les services.

Enfin, l'augmentation légalisée de la précarité (CDD, temps partiel, CNE en France, jobs à 1 euro en Allemagne...), les changements de règles sociales de plus en plus fréquents (en ce qui concerne tant l'indemnisation du chômage que l'âge de la retraite) et l'exigence d'une mobilité spatiale et professionnelle placent les salariés dans l'instabilité. La succession de nombreuses périodes de formation, de chômage, de travail au cours de la vie professionnelle implique des migrations à plus ou moins longues distances. Le choix d'un lieu de vie, l'achat d'une résidence, l'inscription dans un territoire deviennent plus difficiles, risqués, voire impossibles pour certains.

Les modèles socio-productifs et la géographie du sous-développement des Trente Glorieuses persistent encore dans une partie de l'opinion, comme si rien n'avait changé. Les conséquences de la mondialisation n'ayant pas été réellement prises en compte, notamment au niveau politique et sociétal; seuls certains s'y adaptent. Les autres, exclus par la rapidité des changements et l'hypermobilité généralisée, vont grossir les rangs des déçus ou des révoltés. Dans ce contexte, l'absence de stabilité ou de « fixité » économique, à l'échelle d'une génération, voire d'une décennie, pousse beaucoup à l'individualisme et aux rejets de tous les modèles. En tout état de cause, les schémas économiques et géographiques traditionnels, encore largement véhiculés dans l'opinion publique, sont à reconstruire scientifiquement, d'autant qu'apparaissent des espaces productifs d'un autre type.

\section{Les nouveaux espaces productifs}

On ne peut qu'être frappé par la rapidité de la croissance de l'Asie chinoise et indienne qui, bien que s'inspirant des NPI des décennies précédentes, ont brûlé les étapes, notamment pour la technologie. En termes d'activité, ces deux pays étaient, dans les années 80, des pays agricoles à difficulté d'approvisionnement ou des pays industriels débutants. La transformation rapide, constatée 20 ans plus tard, n'apparaît que de manière incomplète 
à certains. En effet, si on fustige au Nord l'agressivité exportatrice de ces puissances, notamment en matière textile, on croît encore, ou l'on veut croire à leur retard technologique. Concernant la Chine, la spécificité du régime fermé sur lui-même et l'idéologie collectiviste ont longtemps caché les transformations rapides vers l'économie de marché entreprises dès la mort de Mao. En outre, la persistance d'une Chine rurale et miséreuse de 600 millions d'habitants masque statistiquement l'extraordinaire croissance du pays et les progrès réalisés. D'autre part, la coexistence d'un régime autoritaire officiellement marxiste avec le capitalisme et la liberté d'entreprendre constitue un modèle nouveau. Pour l'Inde, l'analyse est plus complexe car s'il s'agit d'une «démocratie ». Dans les deux cas, on note une dualité associant croissance et sous-développement, ainsi qu'une accentuation des inégalités internes.

\subsection{La Chine de l'industrie à la technologie}

Depuis l'adhésion de la Chine à l'OMC en 2001, le pays se livre à une véritable guerre économique grâce à une main-d'œuvre bon marché, une monnaie sous-évaluée et un marché intérieur énorme. En 2005, la Chine est devenue la quatrième puissance mondiale en volume de PIB ( $3^{\text {e }}$ en 2007). L'excédent commercial est passé de 32 milliards de dollars en 2004, à 100 en 2005, soit une progression de $213 \%$. Les exportations (respectivement $+28 \%$ et $+18 \%$ en 2005 et 2006) évoluent vers le high-tech (+28\% en 2006). Comme le montrent des écrits contradictoires, l'ampleur du phénomène n'a pas encore été perçue, tant il a été rapide. Pour Aubert et Sillard (2005), l'activité est souvent limitée " aux secteurs industriels de basse technologie, employant une main-d'œuvre peu qualifiée, comme l'habillement-cuir ou le textile dans les années 1995-2000 ». Au contraire, pour Bouinot (2003) la Chine touche, de fait, un nombre croissant de secteurs dans lesquels on ne l'attendait pas.

Certes, la Chine est devenue dans un secteur traditionnel, le premier producteur d'acier avec $31 \%$ du total mondial et une croissance de $24,6 \%$ en 2005. Mais dans l'agroalimentaire, la Chine qui n'exportait quasiment rien, il y a une vingtaine d'années, assure actuellement plus de $50 \%$ de la production mondiale de pommes : elle est devenue le premier pays exportateur avec 774000 tonnes en 2004, dépassant la France et le Chili. Elle assure également $40 \%$ des exportations mondiales de jus. Même s'il s'agit là d'une production de main-d'œuvre (70\% du coût), on assiste à une percée fulgurante.

Mais, la Chine n'entend pas se cantonner aux secteurs de base comme en témoignent différents exemples depuis 2005. En matière aéronautique, outre l'installation d'un centre de recherche Airbus, d'un transfert de technologie... (Fache 2005), un accord prévoit une coopération franco-chinoise 50/50 pour la conception et la production de l'EC 175 destiné au marché intérieur. Airbus qui achète déjà 60 millions de dollars de pièces en Chine prévoit de doubler ses achats à l'horizon 2010. Concernant le train à grande vitesse, sur lequel les pays du Nord fondaient de grands espoirs, la Chine a mis en concurrence l'Allemagne, la France et le Japon puis annoncé début 2006, qu'elle développerait sa propre technologie. En matière informatique, le chinois Lenovo, qui a racheté en 2005 la branche PC de l'américain IBM, fabrique en Chine une nouvelle gamme de PC et de portables vendus sous son nom à l'exportation grâce à une campagne publicitaire commencée en mars 2006. Le fabricant chinois prévoit ainsi s'implanter sur le marché français avec les américains HP et Dell et 
le taïwanais Acer. Lenovo espère sans doute aussi avoir accès à des technologies sensibles, grâce aux usines américaines tombées dans son escarcelle. La disparition des anciens produits Think d'IBM est prévue au profit de la marque Lenovo qui veut aussi occuper une gamme supérieure grâce à un centre de recherche qu'il vient d'ouvrir en Virginie. Dans le domaine nucléaire, la vente de deux réacteurs EPR par Areva s'accompagne de transfert de technologie. Le temps de l'exportation de cuvettes en émail est passé!

\subsection{Le cas de l'Inde}

Le cas de l'Inde, plus discrète, n'en est pas moins parlant quant à la montée du pôle productif asiatique. Derrière l'OPA médiatisée de Mittal dans le secteur de l'acier, l'informatique, les services et la recherche se développent ou s'installent en Inde dans le cadre des transferts Nord-Sud. Les grands de l'informatique américaine investissent certes dans la fabrication de base ou l'assemblage (ordinateurs à bas prix pour ADM), mais aussi dans des activités plus complexes : Microsoft pour les logiciels, Intel pour les microprocesseurs et Cisco pour les équipements de réseaux Internet. Ce sont donc non seulement les fabrications qui sont délocalisées, mais aussi la recherche, jusque-là chasse gardée du Nord. Wipro, (géant indien informatique) a racheté un laboratoire de recherche à SophiaAntipolis. Néanmoins, selon le rapport Nasscom-Mc Kinsey, le manque d'ingénieurs qualifiés pourrait ralentir la croissance en raison d'une hausse des salaires de 25 à $30 \%$ /an. Depuis peu, l'Inde investit discrètement (tableau 2) dans les secteurs porteurs du Nord : 136 acquisitions à l'étranger en 2005, pour 4,3 milliards de dollars.

Tableau 2 : Principaux achats indiens réalisés en 2005/2006

\begin{tabular}{|l|l|l|l|}
\hline Entreprise achetée & Pays d'origine & Activité & Entreprise acheteuse \\
\hline Docpharma & Belgique & Pharmacie générique & Matrix \\
\hline Premier Food & Grande-Bretagne & Thé & Apeejay \\
\hline Thomson & France & Tube cathodique & Videocom \\
\hline Hôtel Pierre & États-Unis & Hôtellerie & Tata \\
\hline Arcelor & Luxembourg & Acier & Mittal \\
\hline
\end{tabular}

Source : Grant Thornton

De même, le transfert des services, impensable il y a encore quelques années, s'accélère (plates-formes téléphoniques, opérations bancaires et gestion des traitements pour les entreprises anglaises, analyse des résultats médicaux et radiologiques pour les Américains). Les entreprises françaises considérées, comme faiblement implantées en Inde, y sont pourtant fortement présentes employant du personnel aussi bien dans le secteur de la fabrication que dans celui de la recherche. En 2006, la mission économique française à Delhi recense déjà 270 sociétés françaises implantées, employant 35000 personnes dans des filiales, des succursales. Tous les secteurs sont concernés. Saint-Gobain fabrique du verre flotté et automobile à Chennai (2 usines); Alsthom, qui emploie déjà 3000 personnes dans l'énergie et les transports, va confier son ingénierie à Infotech; Accor a investi 140 millions d'euros pour introduire les hôtels Ibis en Inde; Alcatel 
projette l'emploi de 2000 ingénieurs recherche et développement; Cap Gemini augmente ses effectifs... Au total, la France emploie déjà en Inde plus de10 000 chercheurs. Le temps de l'exportation des cotonnades est passé!

Ces nouveaux espaces productifs modifient naturellement la géographie économique : rééquilibrage Nord-Sud, libéralisme affiché et protectionnisme déguisé induisent de brutales modifications spatiales. Le schéma Nord-Sud est remis en cause : la hiérarchie économique est bouleversée par la montée de certains espaces et le déclassement des autres.

\section{La recomposition des territoires économiques}

L'hypermobilité et la brutalité des changements évoqués ci-dessus ont pour conséquence un remodelage économico-spatial à différentes échelles.

\subsection{Libéralisme mondialisé et protectionnisme localisé}

Le libéralisme économique et la mondialisation, qui gagnent la totalité du monde après la chute du communisme, sont apparus pour ceux qui les prônaient, notamment les pays de la Triade, comme un moyen de renforcer des situations acquises. Or, si comme espéré les échanges n'ont jamais été aussi nombreux, les profits aussi importants, le consensus libéral se voit limité par le bouleversement de la hiérarchie économique. Autrement dit, l'apparition de nouveaux pays dans le jeu mondial a provoqué des réactions fortes, y compris parmi les tenants les plus acharnés du libéralisme. Elles vont de la volonté à réguler l'économie-monde au protectionnisme assumé.

Derrière les discours officiels sur l'indispensable ouverture des marchés se profilent des limites, sinon un protectionnisme rampant. Les exemples abondent en 2006. Les parlementaires américains ont proposé de taxer les importations chinoises de $27 \%$ jusqu'à une réévaluation conséquente du yuan. La commission de Bruxelles a décidé d'imposer, pour une durée limitée, des mesures anti-dumping en taxant les chaussures chinoises et vietnamiennes à $20 \%$, tandis les producteurs européens (Italie, Portugal, Espagne) réclamaient une taxation à $50 \%$.

$\mathrm{Ce}$ «nationalisme économique » mis en avant par les gouvernements et certains patrons fait fi des réalités. Faut-il rappeler que l'entreprise Arcelor, présentée comme victime d'une OPA inamicale de Mittal en 2006, venait de racheter dans les mêmes conditions une entreprise canadienne ? La guerre fait aussi rage à l'intérieur de la CEE, sans aucun égard pour les principes communautaires. En 2006, face à une OPA hostile de l'Italien Enel, une fusion Gaz de France-Suez, préparée dans l'urgence sous l'égide du ministre de l'économie, visait à la création d'un mégagroupe français. En Espagne, le gouvernement a cherché une parade pour éviter l'OPA de l'Allemand Eon sur le groupe Endesa. Les gouvernements européens, après avoir prôné le libéralisme et l'avoir inscrit dans le projet de constitution, se déchirent entre alliés dès qu'une entreprise étrangère, fut-elle communautaire, essaie de mettre la main sur une entreprise nationale. Le rachat du groupe P.O. par Dubaï Port World, qui plaçait de fait 6 grands ports américains sous gestion des émirats, a provoqué des polémiques, notamment au sein du parti républicain fervent partisan officiel du libéralisme. En la circonstance, une alliance sacrée allant de certains républicains aux démocrates a combattu cette mise sous capitaux étrangers « inamicaux » 
(aussi qualifiés de capitaux « sales ou terroristes » par la presse économique), au point que Dubaï a reculé. La remise en cause des positions hégémoniques anciennes, constitue une limite au libéralisme doctrinal affiché.

Le «nationalisme économique » est un concept difficile à comprendre/défendre par les adhérents à l'OMC, eu égard aux positions antérieures. Il contredit l'idéologie libérale affichée et l'action de tous les gouvernements qui ont œuvré pour l'instauration de la mondialisation : il est vrai qu'ils ne la maîtrisent plus. Ce «nationalisme économique », qui n'a plus concrètement grand sens tant les croisements de capitaux sont déjà importants, relève donc de deux motivations. D'une part, il délivre un discours rassurant à l'électeur / salarié désemparé par les délocalisations. D'autre part, il vise à une éventuelle reprise en main du pouvoir par les gouvernements qui ont perdu leur puissance face à l'entreprise privée et sans frontière. Le seul levier dont disposent encore les gouvernements est celui de la monnaie dont la sous-évaluation permet une meilleure compétitivité. Mais là encore le marché domine et, en Europe, la monnaie commune, qui ne permet pas ces petits jeux, implique au contraire un consensus.

Ce «nationalisme économique » qui concerne aussi le Sud, peut prendre d'autres formes. Le gouvernement indien, partisan du laisser-faire, surveille toutefois certains domaines stratégiques; l'électronique est ouverte sauf en ce qui concerne la défense; la distribution est surveillée pour protéger les emplois du petit commerce. En Chine, où le pouvoir est fort, et où l'état possède encore une grande partie des entreprises, apparaissent des formes subtiles. En matière de cinéma par exemple, la Chine est complètement fermée en dépit d'un marché énorme, sur lequel lorgnent de nombreux étrangers. Une censure et une législation drastiques bloquent la distribution des films étrangers qui ne constituent que $5 \%$ du marché. À la censure morale et politique (pas de critique gouvernementale) s'ajoutent la répartition inégale des recettes (87\% pour la Chine) et le piratage généralisé. Ici politique et économie nationales s'épaulent pour justifier une forme de protectionnisme discret.

\subsection{Une géographie aréolaire du «sous-développement social »}

Parallèlement à la mondialisation définie comme la diffusion spatiale de l'emprise du capitalisme (Carroué 2002), il convient de prendre en compte l'extension du sous-développement sous forme d'îlots dans les pays du Nord. Le concept de sous-développement, défini dans les années 60 (Lacoste 1967), mettait l'accent sur l'insuffisance de la croissance des ressources par rapport à la croissance démographique et la subordination économique à l'égard des pays développés. Quarante ans plus tard, on note une évolution sous l'effet de la mondialisation et de l'explosion migratoire. De nouvelles formes de « sous-développement social » apparaissent ou se développent dans d'autres territoires. Au-delà des moyennes statistiques, les territoires du sous-développement répondent non seulement au schéma traditionnel des inégalités Nord-Sud, mais de plus en plus à celui d'inégalités internes dans états du Nord et ceux du Sud, y compris en période de croissance comme le montre la Chine. À l'exception de l'Afrique, où l'on constate une certaine permanence des situations, des structures productives modernes exportatrices, à capitaux locaux, fleurissent un peu partout dans le Tiers-Monde et avec elles, une classe moyenne (Bejing information 2005). Le magazine Forbes qui dénombrait 476 milliardaires en 2003 et 793 en 2006, note 
la croissance des Russes (33), des Moyens Orientaux (56, Turcs compris), des Indiens (27) plus nombreux que les Japonais... Le tableau 3 montre qu'il s'agit non de rentiers, mais d'hommes d'affaires : la plupart ont fondé leur propre entreprise et atteignent une surface internationale en quelques années

L'enrichissement de la Chine ou de l'Inde, qu'on lit dans la progression du BIP, touche non seulement quelques hommes d'affaires, mais une part de la population qui, pour être relativement faible, n'en est pas moins importante dans l'absolu. Pour Giraud (2002) «il est incontestable, ( ) qu'en Asie un ensemble de pays comprenant 3,2 milliards d'individus, soit plus de la moitié de la population mondiale, est engagé ( ) dans un processus de rattrapage des pays riches, mesuré par une croissance plus rapide de leur revenu par habitant moyen, ce dernier terme, « moyen », étant évidemment essentiel »

Si on admettait que $60 \%$ de la richesse chinoise était détenu en 1998 par $1 \%$ de la population, cela constitue une masse de 13 millions de personnes vivant dans l'opulence, soit la population d'une ville comme Pékin. En fait l'enrichissement de ces nouveaux pays productifs doit attirer l'attention sur les déséquilibres sociaux intérieurs croissants. Depuis l'avènement de Deng Xiaoping, 400 millions de personnes sont sorties de la pauvreté en Chine. Le pays, troisième constructeur mondial, est le nouveau débouché de l'automobile; les villes construisent immeubles modernes et infrastructures à tout va; l'espérance de vie atteint 70 ans contre 77 aux États-Unis; les Jeux Olympiques 2008 auront lieu à Pékin. Mais, dans le même temps, le gouvernement a licencié des millions d'employés; les autorités locales ont confisqué les terres des paysans pour construire des zones d'activité et de l'immobilier spéculatif. La croissance économique chinoise n'est pas répartie spatialement et socialement : emplois, richesses, équipements se concentrent essentiellement à proximité du littoral, notamment entre Shanghai et Pékin, et ne concernent que certaines catégories professionnelles. Selon le rapport 2001 de la banque mondiale, l'indice de Gini, qui mesure l'inégalité interne d'une distribution de revenus (de 0 pour l'égalité complète à 100 pour l'extrême inégalité), était au milieu des années 90 , de 40 en Chine « communiste », contre 33 en France.

Tableau 3 : les milliardaires du Tiers Monde parmi les 25 premiers

\begin{tabular}{|l|l|l|l|l|l|}
\hline Nom & Rang & Pays & $\begin{array}{l}\text { Lieu } \\
\text { de résidence }\end{array}$ & Secteur d'activité & Fonction \\
\hline $\begin{array}{l}\text { Carlos Slim } \\
\text { Helú }\end{array}$ & 3 & Mexique & Mexico & Télécommunications & $\begin{array}{l}\text { homme d'affaires } \\
\text { (Telmex) }\end{array}$ \\
\hline $\begin{array}{l}\text { Lakshmi Mittal } \\
\text { Prince Alwaleed } \\
\text { Ben Talal } \\
\text { Alsaud }\end{array}$ & 5 & Inde & RU & Srabie \\
\hline $\begin{array}{l}\text { Li Ka-shing } \\
\text { saoudite }\end{array}$ & 10 & Ryad & Finances & Steel Company) \\
\hline Azim Premji & 25 & Inde & Hong Kong & commerce & homme d'affaires \\
\hline
\end{tabular}

Source : Forbes 2006 
Dans le même temps, on note en pays développés, y compris dans le groupe du G8, des signes de sous-développement : baisse de l'espérance de vie en Russie, augmentation de l'illettrisme ou du nombre de pauvres en France, incapacité des pouvoirs publics à faire face aux crises (Nouvelle-Orléans).

Tous ces exemples montrent que le schéma Nord-Sud est en mutation : le sous-développement, comme la richesse ne sont plus statiques. Ils ne sont plus caractéristiques de certains pays, de certaines zones géographiques, mais ils sont de plus en plus mobiles et dispersés en archipels (Veltz 2004). Le concept d'a-centralité développé par J. Fache (2007) prend ici tout son sens; ce sont les paramètres exogènes qui dominent. Au-delà des moyennes statistiques nationales, c'est-à-dire spatialisées, on constate que le concept centre-périphérie est devenu essentiellement social. La périphérie ne se présente plus sous forme d'un continuum, mais d'isolats dispersés. Elle est devenue aréolaire (Montagné Villette 2004).

\section{Conclusion}

Ce rééquilibrage économique et social, qui s'inscrit dans un temps court, a des conséquences scientifiques et spatiales profondes. Avec l'hypermobilité, le temps de la conceptualisation a bien du mal à s'accorder au temps économique. Avec la mondialisation, le Tiers-Monde comme le sous-développement social, ne relèvent plus des échelles auxquelles ils ont été pensés. Dans de nombreux cas, la notion est applicable non plus à une zone, à un pays, mais à des régions, des villes, voire des îlots. De même, les périphéries sont non plus de vastes territoires d'un seul tenant, mais des aréoles discontinues. Cependant la situation actuelle peut être de nouveau perturbée. D'une part, la diminution des ressources, et notamment des réserves pétrolières qui permettent des coûts de transport extrêmement bas, peut modifier la donne. D'autre part, les gouvernements, privés d'une part de leur pouvoir par les entreprises, peuvent revenir à un libéralisme atténué qui ralentirait les échanges. Le «nationalisme» et/ou le «patriotisme économique » ressemblent fort à une fermeture des états riches, qui pensaient être les seuls gagnants du libéralisme. L'apparition de nouveaux pôles de richesse peut changer la donne, à moins que ce ne soit la révolte des nouveaux pauvres. En tout état de cause, la géographie économique est à reconstruire.

\section{Sites web}

Bejing information.com site web $2005 \mathrm{~N}^{\circ} 4$ : la classe moyenne définie par les chiffres $6 \mathrm{p}$

FIG 2005 : F.Bost : organisateur de la table ronde : les délocalisations sont-elles fatales?; site web du festival de Saint Dié

Regional Studies (site) Montagné Villette S (2004) Spatial peripheries, social peripheries; in actes du colloque « Europe at the margins »10p 


\section{Références}

Aubert P. Sillard P. (2005 juin) Délocalisations et réduction d'effectifs dans l'industrie française; étude INSEE.

Bouinot J. (2004) Des évolutions dans les comportements spatiaux des entreprises en 2003; Cybergéo avril 2004

Bekouche P. Damette F. (1993) Une grille d'analyse globale de l'emploi. Economie et statistiques $\mathrm{N}^{\circ} 270$, pp37-50

Bekouche P. (1999), De l'industrie à l'industry. Elargir l'acception de l'industrie dans la géographie française ; Géographie, Economie, Société, vol.1, N², Paris, Alternatives Economiques, pp 307-328.

Benko G. (1986) Quelques considérations sur les activités de pointe; BAGF, 1986-4, pp329-339

Bost F., Delapierre M. (2007) Formation des réseaux internationaux de production et reconfiguration des territoires. Economies et Sociétés, série Relations économiques internationales $\mathrm{N}^{\circ} 38$, pp237-254.

Carroué L. (2002) Géographie de la mondialisation; A. Colin, Paris, 256p

Daviet S. (2005) Industrie, culture, territoire; L'Harmattan, Paris, 212p

Dezert B (1986) Les mutations technologiques et le changement industriel; BAGF 1986-3, pp212-215

Fache J (2005) EADS : le territoire émergent d'une firme européenne; L'information géographique $\mathrm{N}^{\circ} 69$, pp132-149.

Fache J (2007) Polarités d'EADS et a-centralité de l'espace; dans Nouvelles échelles des firmes et des réseaux. Un défi pour l'aménagement, sous la direction de Dupuy G. et Géneau de Lamarlière I. Paris, L'Harmattan, 247p

Giraud P.N. (2002) Mondialisation et dynamique des inégalités; Ecole Nationale Supérieure des Mines http:// www.cerna.ensmp.fr 13p

Gorgeux A., Mathieu R. (2000). La filière automobile a-t-elle une gestion locale de la main d'œuvre? dans Ville et emploi. Le territoire au cœur des nouvelles formes de travail. Editions de l'aube. La Tour d'Aigues.

Lebeau A (2005) L'engrenage de la technique : essai sur une menace planétaire. Gallimard, Paris, 272p

Manzagol C. (1980) Logique de l'espace industriel; PUF, Paris, 248p.

Mérenne-Schoumaker B. (1991) La localisation des industries; Nathan, Paris, 192p

Montagné Villette S (1987) L'industrie du prêt-à-porter en France.; Thèse d'Etat, Paris 1, 839p

Montagné Villette S (1990) Le sentier un espace ambigu; Masson, Paris, 144p

Montagné Villette S. (2001) Le secondaire est-il soluble dans le tertiaire ? Annales de géographie $\mathrm{N}^{\circ} 617$, Paris, pp 22-37

Montagné Villette S. (2005) : L'innovation organisationnelle du travail : entre proximité et éloignement ; Cahiers Nantais 62-63, pp 137-147

Montagné Villette S. (2007) Polarités et aménagement dans le chaos du court terme. dans Nouvelles échelles des firmes et des réseaux. Un défi pour l'aménagement, sous la direction de Dupuy G. et Géneau de Lamarlière I. Paris, L'Harmattan, 247p

Levitt S., Dubner J. (2006) Freakonomics, Paris, Denoël, 301p

Rapport remis au Premier Ministre : Besoins de main-d'œuvre et politique migratoire; mars 2006, 146p à paraître à la documentation française.

Perraudin C. et al. (2006) Sous-traiter ou embaucher? 1984-2003 dossier du centre d'études de l'emploi N 78, Marne la Vallée, 34p.

Pottier C. Les multinationales et la mise en concurrence des salariés. Paris, L'Harmattan, 252p.

Roussel J., Assad G., Singaby P., Glogowski E. (2005) : European Supply Chain Trends Influencing the Management; Agenda b. PRTM Paris 15p. prtm.com.

Veltz P. (1996), Mondialisation, villes et territoires, L'économie d'archipel, PUF, réédité en 2004 (col. Quadrige) 